\title{
Automatic Segmentation of Cracked Inclusions in Ductile Alloys
}

\author{
Y. Mao, A. M. Gokhale, J. Harris
}

School of Materials Science and Engineering, Georgia Institute of Technology, Atlanta, GA-30332

Ductile fracture of many industrially important alloys involves cracking of intermetallic inclusions/particles, growth of voids at the cracked particles, and void coalescence. Therefore, quantitative characterization and modeling of the damage due to particle cracking are of interest. In earlier studies, the number fraction of cracked constituent particles has been experimentally measured to quantitatively characterize such damage nucleation [1-3]. However, to the best of authors' knowledge, all earlier experimental studies on characterization of particle cracking involved manual detection of cracked particles. Such measurements are tedious and do not permit large statistical sample sizes due to the practical limitations on time that can be spent on the measurements. Therefore, it is of significant practical interest to develop a digital image analysis based procedure for automatic detection of cracked particles in the microstructural images. The automatic detection of cracked constituent particles is particularly problematic in the wrought Alalloys that also contain $\mathrm{Mg}_{2} \mathrm{Si}$ particles because the gray scale regimes of the cracks overlap those of the $\mathrm{Mg}_{2} \mathrm{Si}$ particles. The objective of this contribution is to report a novel digital image analysis technique that permits automatic detection of cracked inclusions / constituent particles in wrought and cast Al-alloys, so that the measurement can be performed on large statistical samples to obtain robust and precise mean values of the fraction of the cracked particles (and other attributes) with minimal effort. The image analysis technique is presented through its application for detection of cracked constituent particles in A356 cast Al-alloy, and 5086, 6061, and 7075 wrought Al-alloys.

The experiments have been performed on tensile test specimens of A356 cast Al-alloy, and 5086, 6061, and 7075 wrought Al-alloys. These tests have performed at room temperature in the strain-controlled mode at the strain rate of $10^{-4}$. The specimens were cut along a vertical plane containing the applied stress direction, and then mounted and polished using standard metallographic techniques. The digital image analysis procedure involves (1) creation of seamless montage of 64 contiguous microstructural fields using the montage technique developed by Louis and Gokhale [4], (2) detection of the contours of the constituent particles, (3) separation of the $\mathrm{Mg}_{2} \mathrm{Si}$ particles, and (4) identification of the cracked Fe-rich particles using the new image analysis algorithm and their segmentation. Figure 1 depicts the seamless microstructural montage of 64 contiguous fields of view. Note that montage creation is essential to minimize the bias in the measured microstructural attributes due to "edge effects." Note that the montage is a high-resolution large area $\left(\sim 3 \mathrm{~mm}^{2}\right.$ area $)$ image; each microstructural filed of the montage has the resolution of the microstructural window shown in right side of Figure 1. The next step consists of identifying the contours of the boundaries of the constituent particles using the gray scale transitions along the particle boundaries. Once the particle boundaries are detected, the gray scale transitions between the crack and its surroundings are used to identify the cracked particles. Figure 2 shows the cracked Fe-rich constituent particles segmented in this manner. Once the cracked Fe-rich constituent particles are separated from uncracked inclusions, standard automatic image analysis commands can be used to automatically count the number density of cracked particles, their size distribution, size and orientation of microcracks etc. 


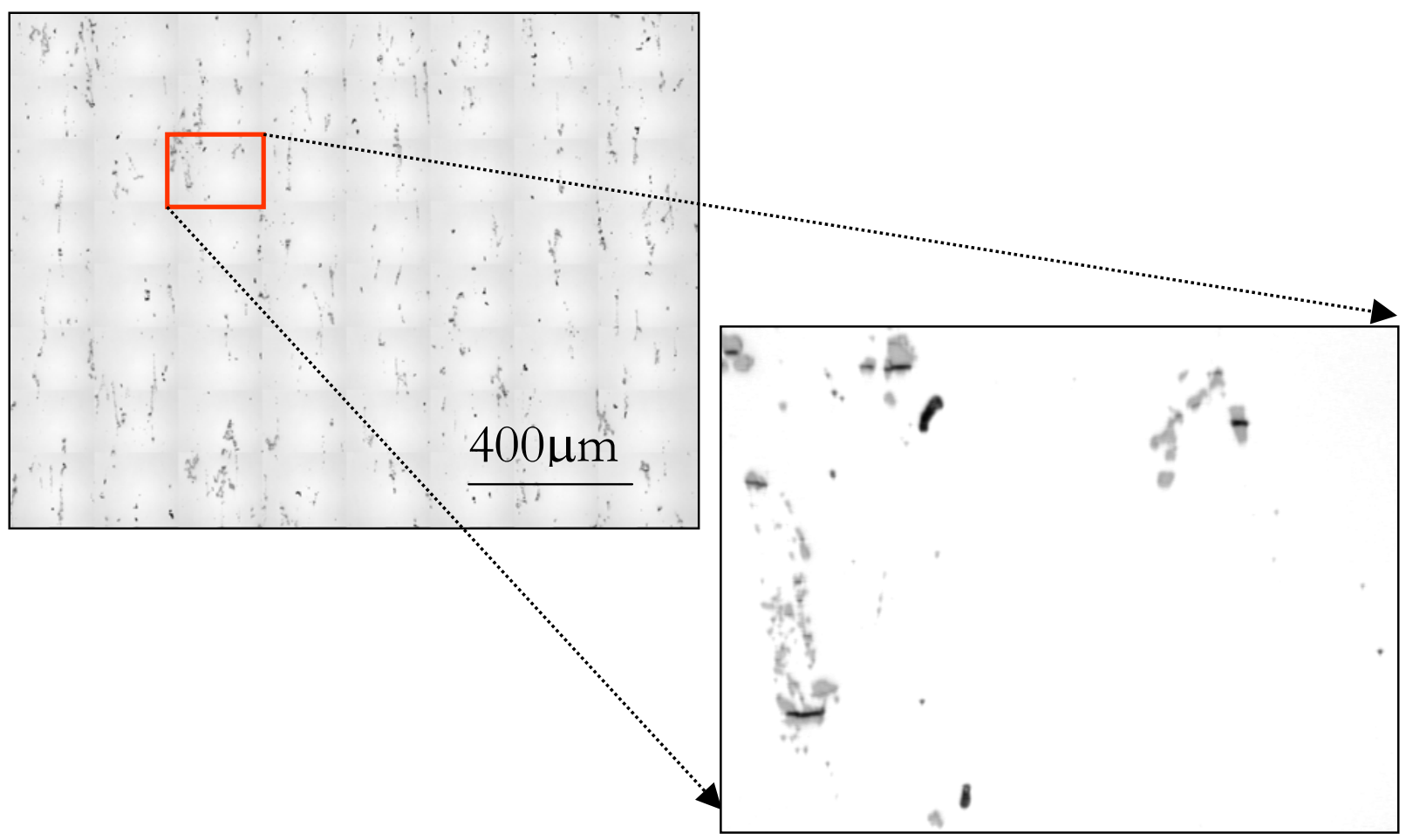

FIG 1: Seamless montage of 64 contiguous microstructural fields captured at the high resolution of the individual field shown on the right.

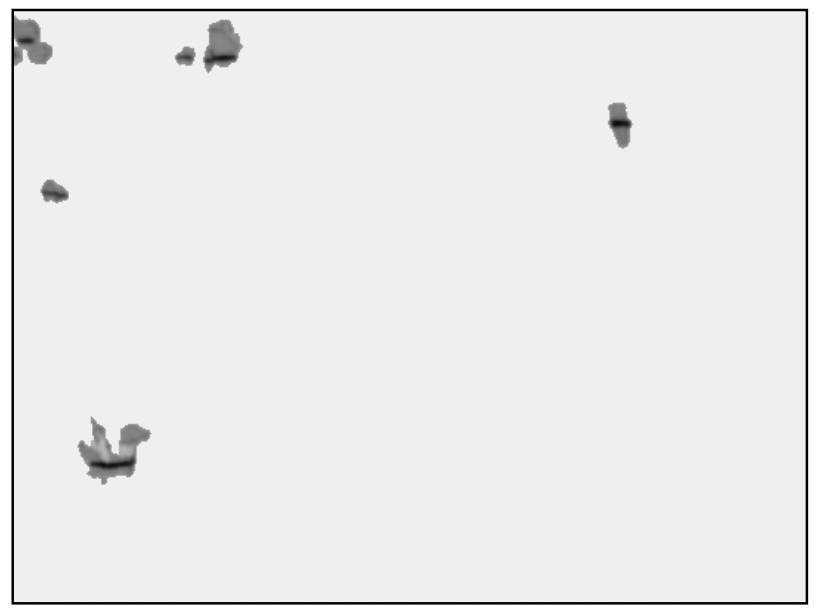

FIG 2: Cracked inclusions separated from the overall inclusion population

\section{References}

[1] M.Dighe, A.Gokhale, and M.Horstemeyer, Metall. \& Mater. Trans., Vol. 31A, PP. 1725, 2000.

[2] H. Agarwal, A.Gokhale, and M.Horstemeyer, Metall. \& Mater. Trans., Vol.33A, PP.3443, 2002.

[3] M.Dighe, A.Gokhale, and M.Horstemeyer, Metall. \& Mater. Trans., Vol. 33A, PP. 555, 2002.

[4] Pascal Louis and A.M. Gokhale, Metall. \& Mater. Trans., Vol. 26A, PP. 1449, 1995.

[5] Acknowledgement: The PROGNOSIS Program of DARPA and National Science Foundation (grant DMR-0404668) supported this work. The financial support is gratefully acknowledged. 